\title{
Photoexcitation induced ultrafast nonthermal amorphization in $\mathrm{Sb}_{2} \mathrm{Te}_{3}$
}

Subodh C. Tiwari, ${ }^{1}$ Rajiv K. Kalia, ${ }^{1}$ Aiichiro Nakano, ${ }^{1}$ Fuyuki Shimojo, ${ }^{2}$ Priya Vashishta, ${ }^{1}$ Paulo Branicio $^{1}$

${ }^{1}$ Collaboratory for Advanced Computing and Simulation, Mork Family Department of Chemical Engineering and Materials Science, University of Southern California, Los Angeles, CA-90007

2 Department of Physics, Kumamoto University, Kumamoto, 860-8555, Japan

\section{Simulation details:}

We perform quantum molecular dynamics simulation ${ }^{1,2}$ with PBE functional and the projector augmented wave (PAW) method. ${ }^{3-5}$ We have generated function for the $5 s, 5 p$ and $5 d$ states of $\mathrm{Sb}$ and Te atoms. The plane-wave cutoff energies are 30 and $250 \mathrm{Ry}$ for the electronic pseudo-wave functions and the pseudo-charge density, respectively in momentum space formalism. The gamma point is used in the Brillouin zone. Interatomic forces are computed quantum mechanically based on the Hellmann-Feynman theorem. The equations of motion are integrated numerically with a time step of 50 a.u. $(\sim 1.20 \mathrm{fs})$. The model used for excited states simulations is composed of $2 \times 3 \times 1$ repeating $\mathrm{Sb}_{2} \mathrm{Te}_{3}$ crystalline cell in an orthorhombic box. $\mathrm{Sb}_{2} \mathrm{Te}_{3}$ is converted to orthorhombic unit cell by taking projection along the [210] direction. Initially, the system is relaxed using a Quasi-Newton method (BFGS algorithm) at 0 GPa pressure. After geometry optimization, the system is relaxed to $300 \mathrm{~K}$ using Nose-Hoover thermostat and barostat for 2.4 ps. $^{6,7}$ Initial excitation is performed by removing electrons from HOMO to HOMO$n$ bands and placing them in LUMO to LUMO $+n$ bands, where $n$ is number of excited bands. Number of excited electrons are twice the number of excited bands. For $n=2.6,5.2,7.5,10.3$, and $12.5 \%$ excitation, we have excited $13,26,38,52,63$ bands. Figure $\mathrm{S} 1$ shows the final structure after excited state evolution for $n=10.3 \%$ and $12.5 \%$. 


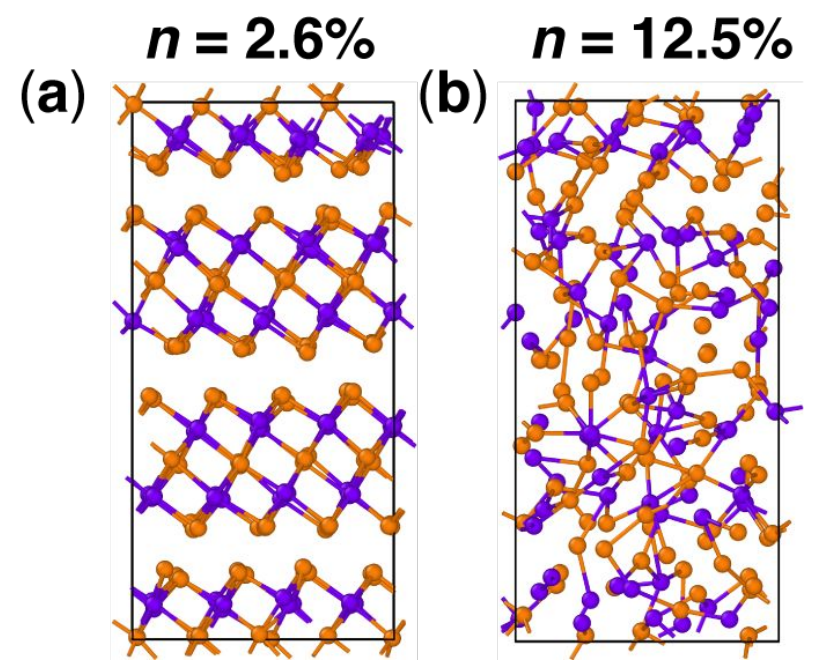

Figure S1: $\mathrm{Sb}_{2} \mathrm{Te}_{3}$ simulation supercell structures after $4.22 \mathrm{ps}$ excited state dynamics with $2.6 \%$, and $12.5 \%$ excited valence electrons, respectively. Yellow and purple spheres correspond to antimony and tellurium atoms, respectively

\section{Electron phonon coupling and its effect of lattice temperature:}

In the nonadiabatic quantum molecular dynamics (NAQMD) simulations, the electron-phonon coupling in fact plays a key role in the relaxation of electronic-excitation energy. In the simulation method used, after the initial excitation, the time evolution of the density matrix is calculated considering a fixed atomic configuration between molecular dynamics (MD) steps. The elements of the density matrix determine the switching probability between the adiabatic states that are expressed by a single Slater determinant as presented by Tully et al. and Duncan et al. ${ }^{8,9}$ The equations for the density matrix are solved using the eigen-energies of the single-electron KohnSham (KS) Hamiltonian and the nonadiabatic coupling (NAC) terms, which are obtained from the current (excited) adiabatic state. ${ }^{8-12}$ These equations are derived by expanding the electronic state $\psi(t)$ at time $t$ in terms of the electronic excited states $\phi_{j}(\mathbf{R}(t))$ in LR-TDDFT corresponding to the atomic configuration $\mathbf{R}(t)$ at time $t$ : 


$$
|\Psi(t)\rangle=\sum_{J} C_{J}^{(I)}(t)\left|\Phi_{J}(\mathbf{R}(t))\right\rangle \quad C_{J}^{(I)}(0)=\delta_{I, J}
$$

The time evolution of the expansion coefficients $C_{J}^{(I)}(t)$ is governed by

$$
\frac{d}{d t} C_{J}^{(I)}(t)=-\sum_{k} C_{k}^{(I)}(t)\left(i \omega_{K} \delta_{J K}+D_{J K}\right)
$$

where the NAC elements are defined as

$$
D_{J K}=\left\langle\Phi_{J}\left|\frac{\partial}{\partial t}\right| \Phi_{K}\right\rangle
$$

The NAC elements are calculated from a pair of excited-state wave functions at consecutive time steps. The surface hopping of electrons is driven by lattice motion (i.e., phonons). Through this electron-phonon interaction, electrons are allowed to relax to ground state from excited states. The excitation energy is converted to heat via electron-phonon coupling, which increases the lattice temperature. In the MD simulations, we are not able to account for the heat dissipation occurring in a macroscopically large experimental sample. Thus, to account for this dissipation, we have implemented a heat sink via a thermostat that controls the temperature.

\section{Estimation of Fluence by Femtosecond-laser pulse:}

We have estimated the fluence of laser pulse for a $250 \mathrm{~nm}$ laser. For a $250 \mathrm{~nm}$ laser pulse, the total energy is $\hbar \omega_{0}=4.78 \mathrm{eV}$, which is much higher than the $\mathrm{Sb}_{2} \mathrm{Te}_{3}$ bandgap, $0.21 \mathrm{eV}$. Thus, we will consider a linear absorption (direct excitation). Using equation given below

$$
N_{e-h}=(1-R) \frac{\alpha_{e f f} F}{\hbar \omega_{0}}
$$

$N_{e-h}$ is the carrier density, $F$ and $\omega_{0}$ the laser fluence and frequency, $R$ the reflectivity, and $\alpha_{e f f}$ the effective absorption coefficient of the sample. $\alpha_{\text {eff }}$ is computed from data provided by Lawar 
et al. ${ }^{13}$ For $n=7.5 \%$, the fluence is $20.3 \mathrm{~mJ} / \mathrm{cm}^{2}$ and total energy density required for amorphization is $20.3 \mathrm{~kJ} / \mathrm{cm}^{3}$.

\section{Estimation of local temperature:}

For $n=12.5 \%$ excitation, we calculate the local temperature by partitioning the system in 12 voxels and estimating the average temperature in each voxel as a function of time, as shown in the figure S2. The 12 voxels of size $\sim 7.4 \times 12.8 \times 5.1 \AA$ are obtained by dividing the simulation supercell of size $\sim 14.75 \times 12.8 \times 30.3 \AA$ by 2,1 , and 6 in the $x, y$, and $z$ directions. From the results shown in figure S2, we can observe that the local temperature in all voxels remain below the melting point $(890 \mathrm{~K})$.

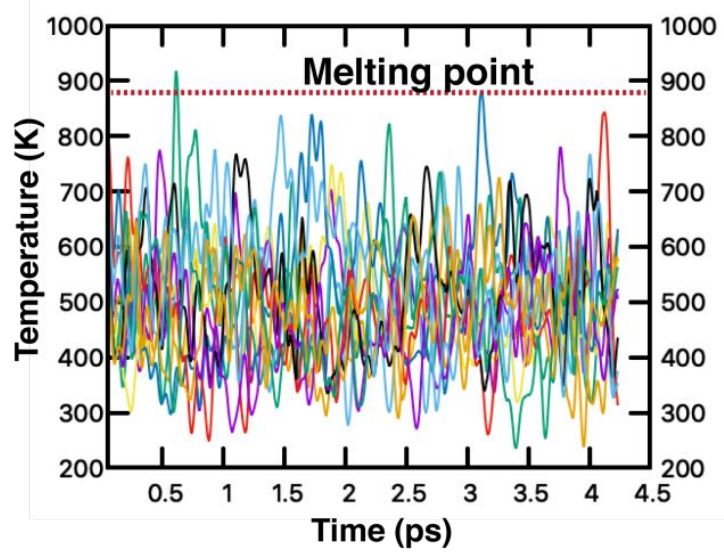

Figure S2: Local temperature of the system with $n=12 \%$ of the valence electrons excited as a function of time. Each line represents the temperature calculated in one of 12 voxels representing the whole volume of the system.

\section{Mean square displacement calculation:}

We have calculated mean square displacement correlation function $\left\langle R^{r}(t)\right\rangle$ over $4.2 \mathrm{ps}$. We have used one initial condition. The mean square displacement (MSD) is averaged over each atom type. Figure S2 show the MSD for Te atoms. Mean square displacement of Te atoms shows similar 
properties as $\mathrm{Sb}$ atoms. However, quantitively mean square displacement is lower than $\mathrm{Sb}$ atom due to higher mass of Te atoms.

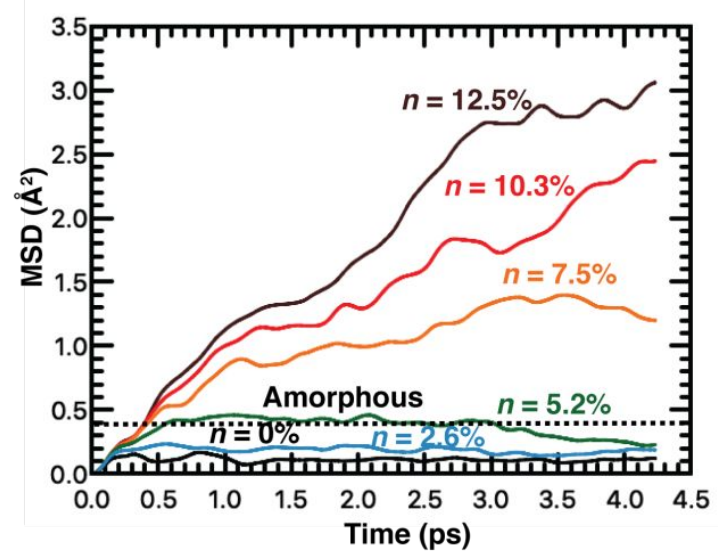

Figure S3: Mean square displacement of Te atoms as a function of time. Cyan, green, orange red and brown curve corresponds to excitation of $n=2.6 \%, 5.2 \%, 7.5 \%, 10.3 \%$ and $12.5 \%$ respectively at temperature $500 \mathrm{~K}$. Black curve corresponds to the adiabatic MD at $500 \mathrm{~K} . \mathrm{n}$ is percentage of excited electron out of the total electrons. Black Dashed curve shows the highest mean square displacement at $800 \mathrm{~K}$.

\section{Partial radial distribution function:}

We have calculated partial radial distribution function of $\mathrm{Sb}-\mathrm{Sb}$, Te-Te and $\mathrm{Sb}-\mathrm{Te}$ pair for $n=0 \%$, $2.6 \%, 5.2 \%, 7.5 \%, 10.3 \%$ and $12.5 \%$. The radial distribution function is average over last $0.2 \mathrm{ps}$ time in NAQMD trajectory. Computed partial radial distribution function is normalized by weighting them with their corresponding concentration. 

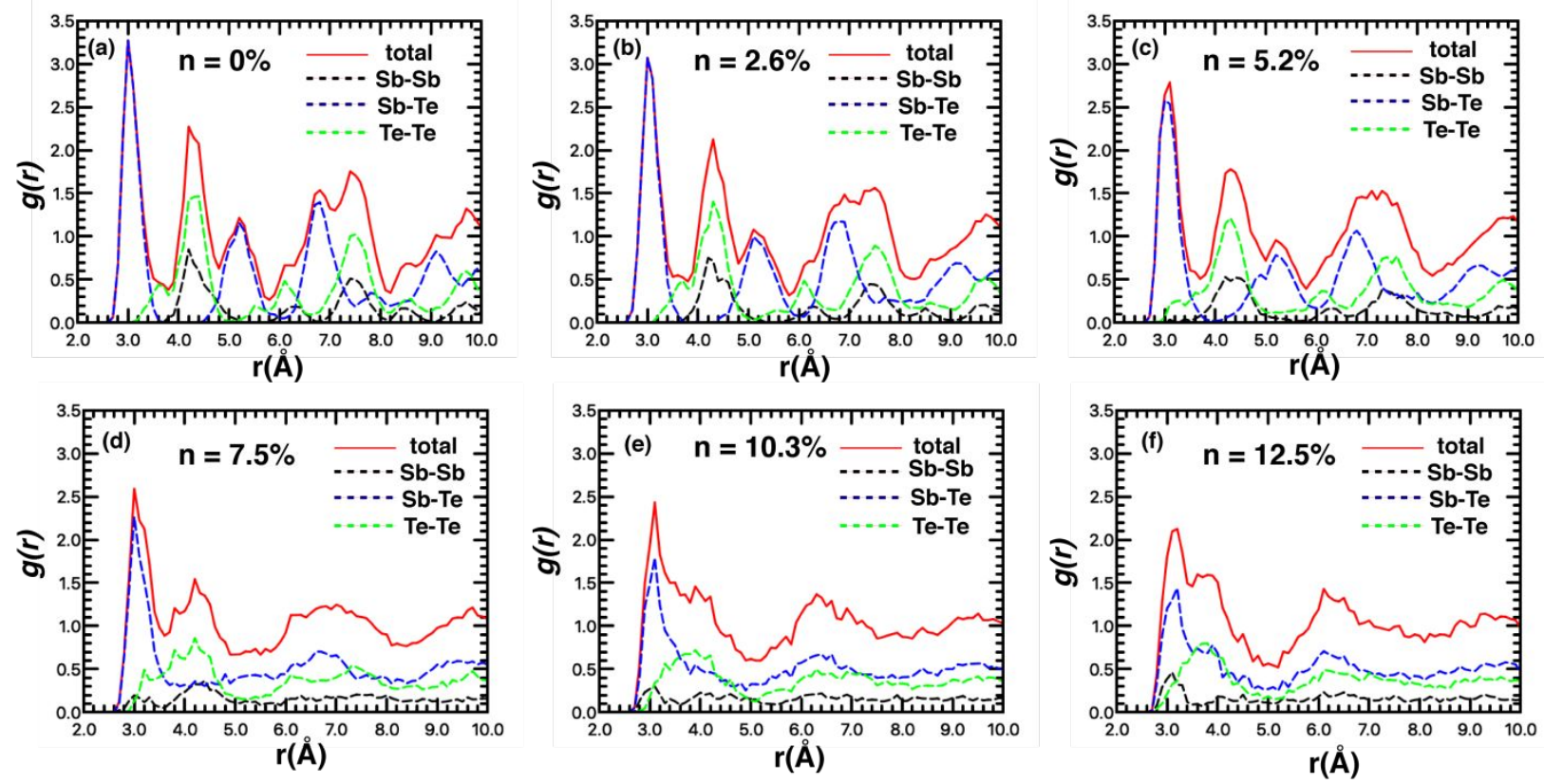

Figure S4: Partial pair correlation function for $n=0 \%, 2.6 \%, 5.2 \%, 7.5 \%, 10.3 \%$ and $12.5 \%$ respectively at temperature $500 \mathrm{~K}$. Total pair correlation function is shown by red solid line SbSb, Sb-Te and Te-Te partial pair correlation function is shown by black, blue and green dashed lines, respectively.

\section{COHP analysis:}

COHP analysis is performed using LOBSTER tool. ${ }^{14-19} \mathrm{We}$ have used a window of -12.5 to $4 \mathrm{eV}$ for calculating COHP analysis. Analysis is performed using pbeVasFit2015 basis set. Sb and Te are projected over $5 s$ and $5 p$ atomic orbitals. After analysis, absolute charge spillage is at $2.24 \%$.

\section{Mulliken charge analysis:}

To quantify the change in local chemical environment and bonds of atoms, we performed bondoverlap population analysis by expanding the electronic wave functions in an atomic-orbital basis set based on the formulation generalized to the PAW method. ${ }^{20} \mathrm{We}$ obtained the gross population 
$Z_{i}(t)$ for each atom and bond-overlap population $O_{i j}(t)$ for a pair of $i^{\text {th }}$ and $j^{\text {th }}$ atoms as a function of time $t$. From $Z_{i}(t)$, we estimate the charge on atom $i$ at time $t$. The $O_{i j}(t)$ gives a semiquantitative estimate of the strength of covalent bonding between atoms. As the atomic-basis orbitals, we used numerical pseudo-atomic orbitals, which were obtained for a chosen atomic energy so that the first node occurs at the desired cutoff radius. The resulting charge spillage, which estimates the error in the expansion, was only $0.15 \%$, indicating the high quality of the basis orbitals. Figure $\mathrm{S} 4$ the bond overlaps for $\mathrm{Sb}-\mathrm{Sb}$, Te-Te and $\mathrm{Sb}-\mathrm{Te}$ pairs, created by averaging all $O_{i j}$ ( $t$ )for same pair type.

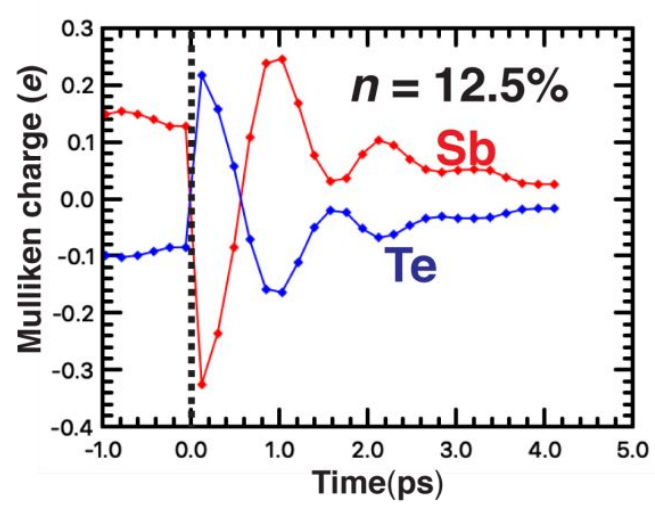

Figure S5: Average Mulliken charge per component as a function of time for $n=12.5 \%$.

\section{Bader Charge analysis:}

Bader charge analysis is performed using bader code. ${ }^{21-24}$ In adiabatic MD simulation, Sb atoms have average charge of $0.6 e^{-}$while Te atoms has $-0.4 e^{-}$. After excitation of $n=5.2 \%$ we observe the average $\mathrm{Sb}$ atom charge decrease to $0.38^{-}$while $\mathrm{Te}-0.253$. Similar changes are observed. Further, increasing excitation lead to depletion of electrons form Te atoms which are transferred to $\mathrm{Sb}$ atoms leading to a decrease in charge on $\mathrm{Sb}$ and increase of charge on Te atoms. 

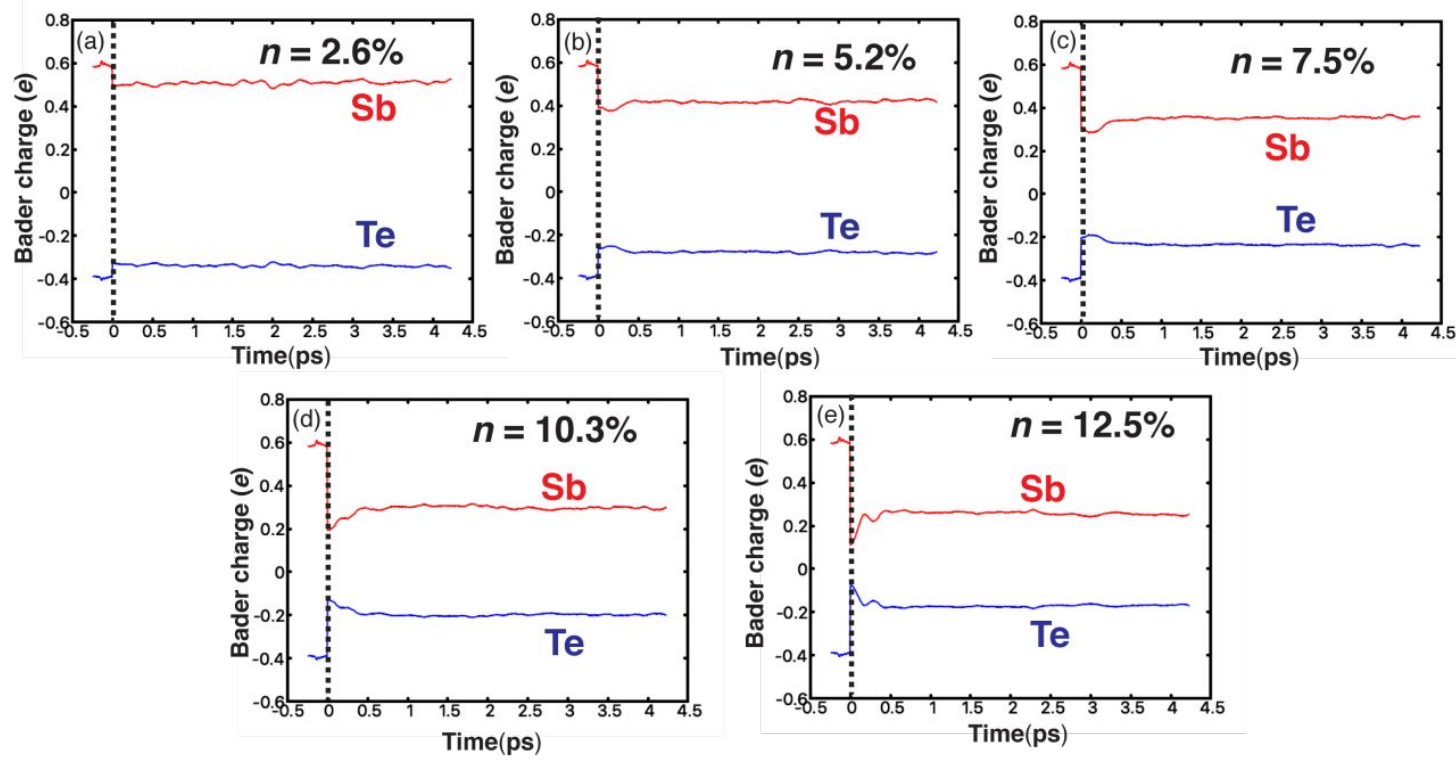

Figure S6: Average Bader charge per component as a function of time for $n=2.6 \% 12.5 \%$.

Mulliken bond population analysis: 
(a)

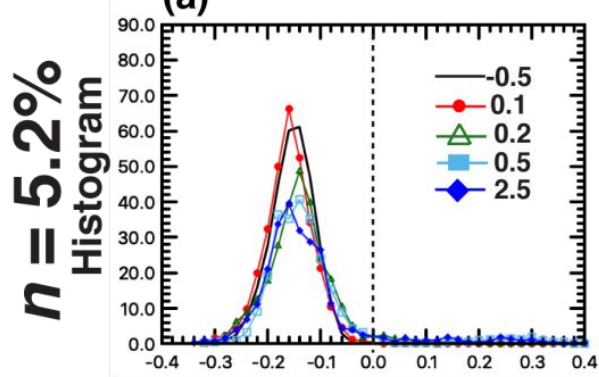

(d)

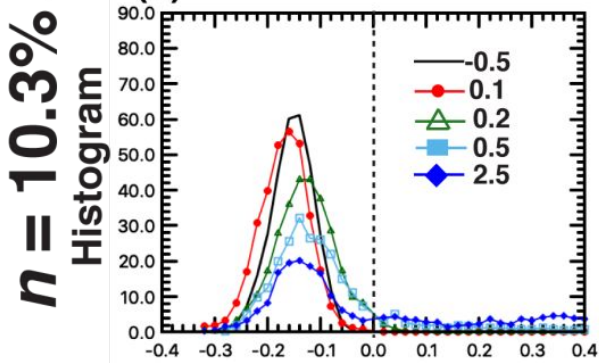

(g)

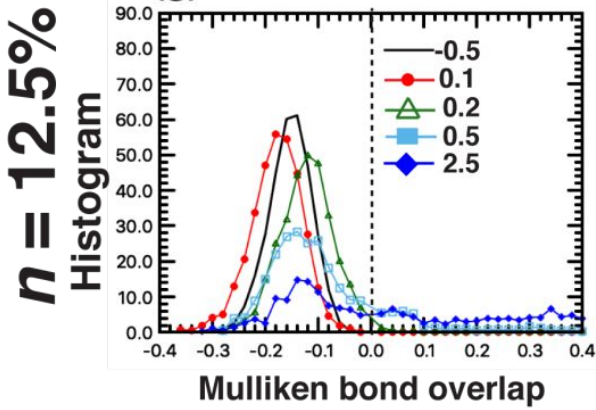

Sb-Te

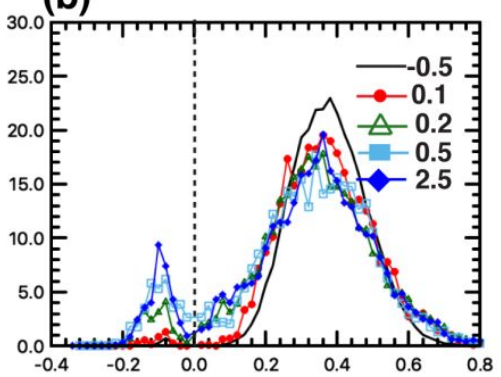

(e)

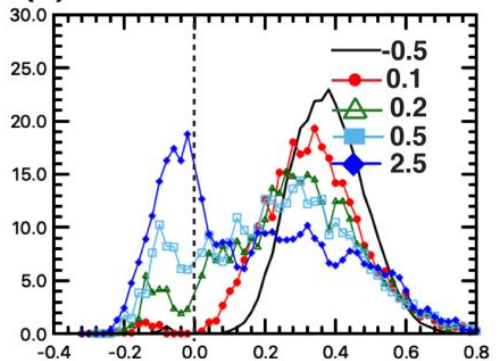

(h)

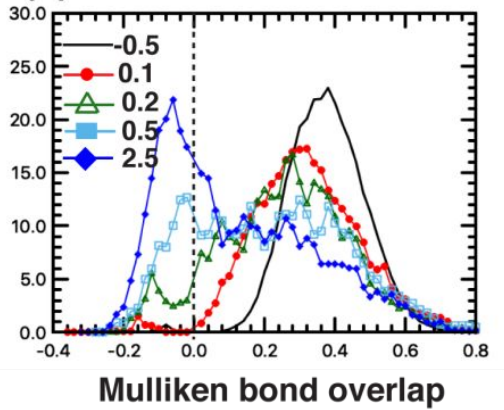

(c) $\mathrm{Te}-\mathrm{Te}$

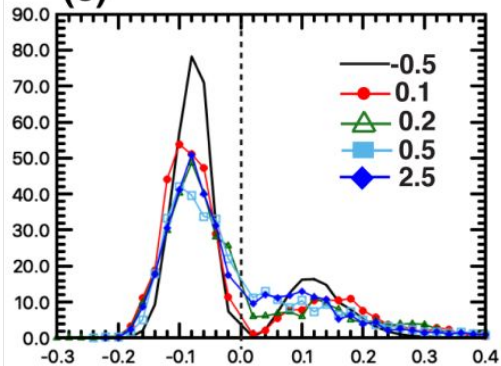

(f)

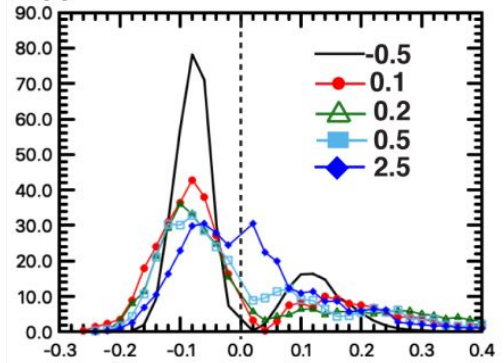

(i)

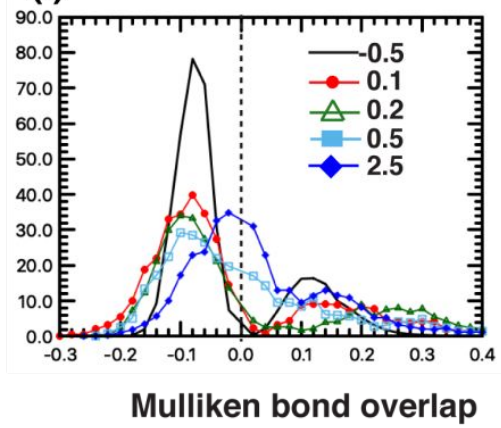

Figure S7: Evolution of Mulliken bond overlap analysis as a function of time for a) Sb-Sb, b) SbTe, and c) Te-Te bond pairs for $n=5.2 \%$. Similarly, d-f) and (g-i) correspond to Sb-Sb, Sb-Te and Te-Te bond pairs for $n=10.3 \%$ and $n=7.5 \%$, respectively. Black color curve corresponds to the $\mathrm{t}=-0.5 \mathrm{ps}$ (before excitation). Red, green, cyan, and blue colors correspond to times $\mathrm{t}=0.01 \mathrm{ps}$, $0.5 \mathrm{ps}, 1.0 \mathrm{ps}$, and $2.5 \mathrm{ps}$ after excitation.

\section{Reference:}

(1) Car, R.; Parrinello, M. Unified Approach for Molecular Dynamics and Density-Functional Theory. Phys. Rev. Lett. 1985, 55, 2471-2474. 
(2) Payne, M. C.; Teter, M. P.; Allan, D. C.; Arias, T. A.; Joannopoulos, J. D. Iterative Minimization Techniques for Ab Initio Total-Energy Calculations: Molecular Dynamics and Conjugate Gradients. Rev. Mod. Phys. 1992, 64, 1045-1097.

(3) Blöchl, P. E. Projector Augmented-Wave Method. Phys. Rev. B 1994, 50, 17953-17979.

(4) Perdew, J. P.; Burke, K.; Ernzerhof, M. Generalized Gradient Approximation Made Simple. Phys. Rev. Lett. 1996, 77, 3865-3868.

(5) Kresse, G.; Joubert, D. From Ultrasoft Pseudopotentials to the Projector Augmented Wave Method. Phys. Rev. B 1999, 59, 1758.

(6) Nosé, S. A Unified Formulation of the Constant Temperature Molecular Dynamics Methods. J. Chem. Phys. 1984, 81, 511-519.

(7) Martyna, G. J.; Tuckerman, M. E.; Tobias, D. J.; Klein, M. L. Explicit Reversible Integrators for Extended Systems Dynamics. Mol. Phys. 1996, 87, 1117-1157.

(8) Duncan, W. R.; Craig, C. F.; Prezhdo, O. V. Time-Domain Ab Initio Study of Charge Relaxation and Recombination in Dye-Sensitized TiO2. J. Am. Chem. Soc. 2007, 129, 8528-8543.

(9) Tully, J. C. Molecular Dynamics with Electronic Transitions. J. Chem. Phys. 1990, 93, $1061-1071$.

(10) Jaeger, H. M.; Fischer, S.; Prezhdo, O. V. Decoherence-Induced Surface Hopping. J. Chem. Phys. 2012, 137.

(11) Casida, M. E. Recent Advances in Density Functional Methods; Chong, D. P., Ed.; Recent Advances in Computational Chemistry; World Scientific Publishing Co. Pte. Ltd., 1995.

(12) Shimojo, F.; Ohmura, S.; Mou, W.; Kalia, R. K.; Nakano, A.; Vashishta, P. Large Nonadiabatic Quantum Molecular Dynamics Simulations on Parallel Computers. Comput. 
Phys. Commun. 2013, 184, 1-8.

(13) Lawal, A.; Shaari, A.; Ahmed, R.; Jarkoni, N. Sb2Te3 Crystal a Potential Absorber Material for Broadband Photodetector: A First-Principles Study. Results Phys. 2017, 7, $2302-2310$.

(14) Maintz, S.; Deringer, V. L.; Tchougréeff, A. L.; Dronskowski, R. LOBSTER: A Tool to Extract Chemical Bonding from Plane-Wave Based DFT. J. Comput. Chem. 2016, 37, $1030-1035$.

(15) Nelson, R.; Ertural, C.; George, J.; Deringer, V. L.; Hautier, G.; Dronskowski, R. LOBSTER: Local Orbital Projections, Atomic Charges, and Chemical-Bonding Analysis from Projector-Augmented-Wave-Based Density-Functional Theory. J. Comput. Chem. 2020, 41, 1931-1940.

(16) Dronskowski, R.; Bloechl, P. E.; Blöchl, P. E. Crystal Orbital Hamilton Populations (COHP). Energy-Resolved Visualization of Chemical Bonding in Solids Based on Density-Functional Calculations. J. Phys. Chem. 1993, 97, 8617-8624.

(17) Deringer, V. L.; Tchougréeff, A. L.; Dronskowski, R. Crystal Orbital Hamilton Population (COHP) Analysis as Projected from Plane-Wave Basis Sets. J. Phys. Chem. A 2011, 115, $5461-5466$.

(18) Maintz, S.; Deringer, V. L.; Tchougréeff, A. L.; Dronskowski, R. Analytic Projection from Plane-Wave and PAW Wavefunctions and Application to Chemical-Bonding Analysis in Solids. J. Comput. Chem. 2013, 34, 2557-2567.

(19) Maintz, S.; Esser, M.; Dronskowski, R. Efficient Rotation of Local Basis Functions Using Real Spherical Harmonics. Acta Phys. Pol. B 2016, 47, 1165-1175.

(20) Segall, M. D.; Shah, R.; Pickard, C. J.; Payne, M. C. Population Analysis of Plane-Wave 
Electronic Structure Calculations of Bulk Materials. Phys. Rev. B 1996, 54, 16317-16320.

(21) Sanville, E.; Kenny, S. D.; Smith, R.; Henkelman, G. Improved Grid-Based Algorithm for Bader Charge Allocation. J. Comput. Chem. 2007, 28, 899-908.

(22) Tang, W.; Sanville, E.; Henkelman, G. A Grid-Based Bader Analysis Algorithm without Lattice Bias. J. Phys. Condens. Matter 2009, 21.

(23) Henkelman, G.; Arnaldsson, A.; Jónsson, H. A Fast and Robust Algorithm for Bader Decomposition of Charge Density. Comput. Mater. Sci. 2006, 36, 354-360.

(24) Yu, M.; Trinkle, D. R. Accurate and Efficient Algorithm for Bader Charge Integration. $J$. Chem. Phys. 2011, 134, 1-8. 